\title{
The importance and challenges of developing a pharmacogenetics test for hypertension
}

\author{
Eric M Snyder ${ }^{1}$, Eli F Kelley², Ryan Sprissler ${ }^{1,3}$ \& Thomas P Olson*,1,4 \\ ${ }^{1}$ Geneticure, Inc., Rochester, MN 55902, USA \\ ${ }^{2}$ School of Kinesiology, University of Minnesota, Minneapolis, MN 55455, USA \\ ${ }^{3}$ University of Arizona Genomics Core, Tucson, Arizona, AZ 85721 USA \\ ${ }^{4}$ Division of Cardiovascular Diseases, Mayo Clinic College of Medicine, Rochester, MN 55905, USA \\ *Author for correspondence: olson.thomas2@mayo.edu \\ "The promise of personalized medicine in hypertension has been speculated upon since late
1990s"
}

First draft submitted: 15 April 2019; Accepted for publication: 26 April 2019; Published online: 28 May 2019

Keywords: hypertension $\bullet$ personalized medicine $\bullet$ pharmacogenetics

The clinical effects of prolonged hypertension are well established and include increased risk of cerebrovascular disease, myocardial infarction (MI) and kidney damage. Many scientists have made significant contributions to the academic understanding of pharmacogenomics in hypertension from genome-wide association studies (GWAS) to individual patient-guided response using SNPs [1-3]. While there is hope for the use of pharmacogenetics to guide therapy in hypertension, widespread adoption has yet to be realized.

For many clinicians, one must first 'prove' that, hypertension is heritable; the response to existing therapy is not uniform; and there is an inherent problem with layering medications that are not effective. First, the heritability of hypertension has been studied, in detail, and is estimated to be approximately $50 \%$. Importantly, $55 \%$ of monozygous twins share hypertensive traits, compared with $40 \%$ of dizygous twins, and $20 \%$ nontwin sibling pairs $[4,5]$. Second, there is a well-established (and dramatically underappreciated) bell-curve response to common hypertension therapies with the blood pressure (BP) lowering effect of medications averaging reductions of approximately $8 \mathrm{mmHg}$ beyond placebo; which is less than most assume [6]. Third, the layering of noneffective therapies becomes progressively less effective due to the known increase in medication nonadherence of $>70 \%$ for each medication added to a patient's clinical care; which can be particularly worrisome in a disease state in which medication compliance is already a well-documented problem [7]. Further, layering of ineffective medications can be problematic because each of the common BP medication classes has well-established side effects, many of which can be serious.

Despite hundreds of peer-reviewed papers demonstrating that genetically guided therapy leads to improvements, or differences, in BP, the use of genetics to guide hypertension treatment is not yet common. Given the integrative physiology of hypertension, both within and between organ systems, the development of a pharmacogenetic panel for selection of one therapy over another is particularly challenging. A reason for this difficulty is that common variants of drug metabolizing enzymes (DME) have little effect on BP as an outcome variable. Historically, DME studies tend to be more common and well controlled in the field of precision medicine. This makes sense given the logical nature of DME on outcomes and that experts in the fields of drug transport and DME were among the first to generate quality research in pharmacogenomics. Additionally, DME variants have been appropriately vetted by groups of experts, including the Clinical Pharmacogenetics Implementation Consortium (CPIC), who then provide guidance for clinicians and research teams. An example of the dichotomy in hypertension pharmacogenomic research is that variants of the CYP2D6 gene have a dramatic effect on plasma drug levels of $\beta$-blockers, but despite this, $\mathrm{BP}$ as an outcome variable is not affected in the majority of studies [8,9]. Interestingly, CYP2D6 is listed by the US FDA on the label for many $\beta$-blockers and has been given a $2 \mathrm{~A}$ evidence level by CPIC, although this appears 
specific to plasma levels and bradycardia, rather than BP. Because of this FDA labeling, and guidance by CPIC, BP is listed as one of many conditions on panels that have a focus on DMEs. However, hypertension is complex and, as mentioned previously, $\mathrm{BP}$ as an outcome variable has not been consistently shown to be affected by CYP2D6 variants alone. In contrast, variants of the adrenergic receptors, among others, have been shown to have a dramatic effect on BP when a patient has a functional genotype and is on a genetically guided target therapy, but there remains no consensus statement on these more functional genotypes. Therefore, there can be an important contribution to this field by smaller groups of academics with more diverse expertise to establish pharmacogenetic panels that include functional variants in the sympathetic nervous, kidney and renin-angiotensin-aldosterone systems, along with DME genotypes that may be important for BP response to therapy.

The promise of genetically guided treatment has been discussed for more than two decades. However, one of the reasons for relatively slow clinical adoption of existing pharmacogenomic tests in the medical setting, and an area of critique, is a lack of evidence demonstrating that a specific pharmacogenetic test is of benefit to the patients, beyond the standard of care [10]. Establishing this evidence is a relatively high bar, but rightfully so given that these tests are designed for use in clinical care. Companies and academic medical centers that implement pharmacogenetic panels to guide therapy must generate evidence that their specific panel is of benefit to the patients. More often than not, companies and academic centers have simply generated a pharmacogenetic panel from a number of existing peer-reviewed publications, and immediately began use and/or commercialization without demonstrating efficacy of the panel in clinical trials, which has led to some resistance by the clinical community. Adoption would be much more robust if those developing personalized medicine tests demonstrated: how and why the genotypes in their panel were selected (through peer review); how the panel as a whole operates on patients with the target disease; and how the panel compares with the currently established standard of care using a blinded randomized control design. This is particularly important in hypertension given the number of genes that are known to be associated with BP. Moreover, until whole genome sequencing and clinician understanding of whole genome sequencing is widespread, there needs to be transparent criteria for the genotypes selected that include allelic frequency (common and generalizable) and effect size (clinically meaningful) [11].

Given the many different genes/genotypes associated with BP regulation, it is important that care is taken when establishing a pharmacogenetics panel for hypertension. GWAS data are important, but selection of genotypes that have been confirmed beyond GWAS, in a priori genetically guided clinical trials should be a focus. Additionally, given the integrative nature of BP regulation, along with the well-established mechanisms of action for common hypertension therapies, candidate gene approaches can be applied. There are good examples of both GWASinitiated BP pharmacotherapy response, and candidate gene selection within hypertension pharmacogenetics. For instance, genetic variation of $\alpha$-adducin (rs4961) has been shown to be associated with hypertension in GWAS and functionally important in a priori trials [12]. Specifically, the T allele of $\alpha$-adducin at position 460 has been shown to be associated with a greater drop in BP with thiazide-like diuretic treatment. Further, research has shown that a patient with the T allele of $\alpha$-adducin who achieves BP control with a diuretic is less likely to have an MI, when compared with patients achieving BP control with a different BP pharmacotherapy, although these results seem mixed $[13,14]$. This is an example of a genotype that was identified though GWAS, confirmed through mechanistic $a$ priori research, and clinical events beyond $\mathrm{BP}$ control are being established. In contrast, the $\beta_{1}$-adrenergic receptor $\left(\beta_{1} \mathrm{AR}\right)$ is a candidate gene because the $\beta_{1} \mathrm{AR}$ makes up $95 \%$ of the SA node (controlling heart rate), $80 \%$ of the ventricular wall (controlling ventricular contractility) and $70 \%$ of the atrial wall. Additionally, $\beta$-blockade is a known effective therapy for the treatment of hypertension. Two common variants of the gene that encodes the $\beta_{1} \operatorname{AR}(A D R B 1$, rs 1801252 and 1801253) have been shown to have a dramatic effect on $\beta$-blocker responsiveness, particularly when considered in combination [15]. Also, similar to $\alpha$-adducin noted above, targeted treatment with a $\beta$-blocker for the functional variant of the $A D R B 1$ at position 49 and 389 leads to reduced risk for MI, whereas treatment with a $\beta$-blocker in the nonfunctional variant (Gly49) has been shown to lead to a slight increased risk of major adverse cardiovascular events, including stroke [16]. This latter point is extremely important as $\beta$-blockade has been relegated to second-line BP therapy. This second-line status is due to a slightly increased risk for stroke, when compared with other BP therapies, although it is clear that there is a genotype effect by which targeted treatment is beneficial for one genotype group and possibly detrimental for another. This is an example of a successful candidate-gene approach that, again, takes into consideration clinical outcomes beyond BP.

Although the majority of the work leading to the current promise of making pharmacogenetics for hypertension a possibility has rested in academia, it is apparent that commercialization may play an important role in widespread adoption. There is a great deal of cost in gathering the necessary evidence for any medical test and arguably more 
so in the area of precision medicine, given the relative novelty. It is likely that private-public partnerships will be integral for adoption. Private companies that specialize in precision medicine, with the ability to share the cost and time burden of filing intellectual property, generating clinical data, developing software and coordinating integration into the electronic medical record, can help to make pharmacogenetics in hypertension a reality. Like many commercialization attempts in medicine, there is some general 'push-back' on the commercialization of pharmacogenetic tests, due to the feeling of ownership of this field within academia. However, given the pace of academia, finding a balanced relationship between academia and biotech firms is needed.

The promise of personalized medicine in hypertension has been speculated upon since late 1990s [17]. Limitations to adoption include the relatively large number of genetic factors that regulate BP, a lack of understanding of personalized medicine or genetics by some clinicians, and resistance by clinicians who may insist upon current standard of care, even in the face of emerging data. These roadblocks to adoption can be overcome, particularly in the commercialization stage, with a respect for the practice of medicine. Specifically, a test may determine that one hypertension drug may be ideal for a patient, based on genetics, but other co-morbidities need to be considered, so a clinician should always be involved in the interpretation of this information. This must always be coupled with a solid scientific foundation and publication strategy. Ideally, prior to commercialization or adoption, a hypertension panel should be tested in multiple controlled trials and allow for modification of the panel and retesting as new genotypes are found to be important.

For more than two decades detailed scientific work has been completed on the genetics and pharmacogentics of hypertension. Hypertension pharmacotherapy response is variable, with a response rate of about half of the population for a given drug, and with blood pressure reductions averaging approximately $8 \mathrm{mmHg}$ beyond placebo. Despite this, the current standard of care in hypertension therapy is to treat patients without attention to genetic variants. The purpose of this editorial is to discuss the challenges and opportunities for the development of a multi-gene panel that may predict pharmacotherapy response in patients with hypertension.

\section{Financial \& competing interests disclosure}

EM Snyder, TP Olson and R Sprissler have significant financial interest in Geneticure Inc., which has developed multigene panels for blood pressure prescribing using pharmacogenetics. The authors have no other relevant affiliations or financial involvement with any organization or entity with a financial interest in or financial conflict with the subject matter or materials discussed in the manuscript apart from those disclosed.

No writing assistance was utilized in the production of this manuscript.

\section{References}

1. Bray MS, Krushkal J, Li L et al. Positional genomic analysis identifies the beta(2)-adrenergic receptor gene as a susceptibility locus for human hypertension. Circulation 101(25), 2877-82 (2000).

2. Chapman AB, Schwartz GL, Boerwinkle E, Turner ST. Predictors of antihypertensive response to a standard dose of hydrochlorothiazide for essential hypertension. Kidney Int. 61(3), 1047-55 (2002).

3. Hiltunen TP, Donner KM, Sarin AP et al. Pharmacogenomics of hypertension: a genome-wide, placebo-controlled cross-over study, using four classes of antihypertensive drugs. J. Am. Heart Assoc. 4(1), e001521 (2015).

4. Hottenga JJ, Boomsma DI, Kupper N et al. Heritability and stability of resting blood pressure. Twin Res. Hum. Genet. 8(5), 499-508 (2005).

5. Snieder $\mathrm{H}$ et al. Heritability of central systolic pressure augmentation: a twin study. Hypertension 35(2), 574-579 (2000).

6. Turnbull F, Neal B, Algert C et al. Effects of different blood pressure-lowering regimens on major cardiovascular events in individuals with and without diabetes mellitus: results of prospectively designed overviews of randomized trials. Arch. Intern. Med. 165(12), 1410-1419 (2005).

7. Gupta P, Patel P, Štrauch B et al. Risk factors for nonadherence to antihypertensive treatment. Hypertension 69(6), 1113-1120 (2017).

8. Lefebvre J, Poirier L, Poirier P, Turgeon J, Lacourciere Y. The influence of CYP2D6 phenotype on the clinical response of nebivolol in patients with essential hypertension. Br. J. Clin. Pharmacol. 63(5), 575-582 (2007).

9. Zineh I, Beitelshees AL, Gaedigk A et al. Pharmacokinetics and CYP2D6 genotypes do not predict metoprolol adverse events or efficacy in hypertension. Clin. Pharmacol. Ther. 76(6), 536-544 (2004).

10. Pletcher MJ, McCulloch CE. The challenges of generating evidence to support precision medicine. JAMA Intern. Med. 177(4), 561-562 (2017).

11. Phelps PK, Kelley EF, Walla DM et al. Relationship between a weighted multi-gene algorithm and blood pressure control in hypertension. J. Clin. Med. 8(3), (2019). 
12. Sanada H, Jones JE, Jose PA. Genetics of salt-sensitive hypertension. Curr. Hypertens. Rep. 13(1), 55-66 (2011).

13. van Wieren-de Wijer DB, Maitland-van der Zee AH, de Boer A et al. Interaction between the Gly460Trp alpha-adducin gene variant and diuretics on the risk of myocardial infarction. J. Hypertens. 27(1), 61-68 (2009).

14. Psaty BM, Smith NL, Heckbert SR et al. Diuretic therapy, the alpha-adducin gene variant, and the risk of myocardial infarction or stroke in persons with treated hypertension. JAMA 287(13), 1680-1689 (2002).

15. Johnson JA, Zineh I, Puckett BJ, McGorray SP, Yarandi HN, Pauly DF. Beta 1-adrenergic receptor polymorphisms and antihypertensive response to metoprolol. Clin. Pharmacol. Ther. 74(1), 44-52 (2003).

16. Magvanjav O, McDonough CW, Gong Y et al. Pharmacogenetic associations of beta1-adrenergic receptor polymorphisms with cardiovascular outcomes in the SPS3 trial (secondary prevention of small subcortical strokes). Stroke 48(5), 1337-1343 (2017).

17. Turner ST, Schwartz GL, Boerwinkle E. Personalized medicine for high blood pressure. Hypertension 50(1), 1-5 (2007). 